УДК 517.5

A. Kowalski, I. I. Marchenko

\title{
ON THE MAXIMUM POINTS AND DEVIATIONS OF MEROMORPHIC MINIMAL SURFACES
}

\begin{abstract}
A. Kowalski, I. I. Marchenko. On the maximum points and deviations of meromorphic minimal surfaces, Mat. Stud. 46 (2016), 137-151.

We provide an upper estimate of the magnitude of deviation for a meromorphic minimal surface of finite lower order. The estimate is given in terms of the number of separated maximum points of the norm of the surface. The examples showing that this estimate is sharp are also presented.
\end{abstract}

1. Introduction. Let us consider in $\mathbb{R}^{3}$ a two-dimensional regular space

$$
S=\left\{\mathbf{x}=\left(x_{1}, x_{2}, x_{3}\right) \in \mathbb{R}^{3}: x_{j}=x_{j}(u, v), j=1,2,3,(u, v) \in D \subset \mathbb{R}^{2}\right\},
$$

where $x_{j}(u, v), j=1,2,3$, are twice continuously differentiable real-valued functions for $(u, v) \in D \subset \mathbb{R}^{2}$. The surface $S$ is called minimal if the mean curvature of $S$ vanishes on all points on the surface $([4])$. It is clear that every two-dimensional regular minimal surface can be given in terms of isothermal parameters, i.e. the coefficients of the first fundamental form of the surface $S$ satisfy the condition ([18], [17])

$$
E(u, v)=G(u, v), F(u, v)=0
$$

where

$$
E=\left\|\mathbf{x}_{u}\right\|^{2}=\sum_{j=1}^{3}\left(\frac{\partial x_{j}}{\partial u}\right)^{2}, \quad F=\left(\mathbf{x}_{u}, \mathbf{x}_{v}\right)=\sum_{j=1}^{3} \frac{\partial x_{j}}{\partial u} \frac{\partial x_{j}}{\partial v}, \quad G=\left\|\mathbf{x}_{v}\right\|^{2}=\sum_{j=1}^{3}\left(\frac{\partial x_{j}}{\partial v}\right)^{2},
$$

and $\mathbf{x}(u, v)=\left(x_{1}(u, v), x_{2}(u, v), x_{3}(u, v)\right)$. A necessary and sufficient condition for a regular surface $S$, given in terms of isothermal parameters, to be minimal is that the coordinate functions $x_{j}(u, v)$ are harmonic ([4], [18]). For this reason we shall assume that the minimal surface $S$ is given by (1) in terms of isothermal parameters and the coordinate functions $x_{j}(u, v), j=1,2,3$, are harmonic for $u^{2}+v^{2}<\infty$, except for the poles. The minimal surface, which fulfills that condition is called a meromorphic minimal surface (m.m.s.) [2].

In the years 1960-1970 E. F. Beckenbach and collaborators generalized the original Nevanlinna theory by introducing the theory of meromorphic minimal surfaces ([2]). We just remind the main definitions and results of this theory.

2010 Mathematics Subject Classification: 30D35, 53A10.

Keywords: meromorphic minimal surfaces; maximum modulus point; subharmonic function; Nevanlinna theory.

doi:10.15330/ms.46.2.137-151

(C) A. Kowalski, I. I. Marchenko, 2016 
Let $S$ be the meromorphic minimal surface given by (1) for the functions $\left\{x_{j}(z)\right\}_{j=1}^{3}$, which are meromorphic harmonic in $\mathbb{C}(z=u+i v)$. Let the point $z_{0}=u_{0}+i v_{0}$ be an $a_{j}$-point of order $p_{j}$ for a harmonic function $x_{j}(z), j=1,2,3([2])$. We say that the point $\mathbf{a}=\left(a_{1}, a_{2}, a_{3}\right)$ is an $\boldsymbol{a}$-point of the order $t=\min \left(p_{1}, p_{2}, p_{3}\right)$ of m.m.s. $S$ at the point $z_{0}$.

We say that a m.m.s. $S$ has a pole at $z_{0}=u_{0}+i v_{0}$, if at least one of the coordinate function $x_{j}(z), j=1,2,3$, has a pole at $z_{0}$. Moreover if $l_{j}$ is the order of the pole of $x_{j}(z), j=1,2,3$, then $l=\min \left(l_{1}, l_{2}, l_{3}\right)$ is called an order of the pole of a m.m.s. at $z_{0}$.

The a-points and the poles of a m.m.s. are isolated ([2]).

For m.m.s. $S$ E. F. Beckenbach defined three functions characterizing a m.m.s.: a proximity function $m(r, \mathbf{a}, S)$ of $S$, a a-points counting function $N(r, \mathbf{a}, S)$ of $S$ and a visibility function $H(r, \mathbf{a}, S)$, which are defined like below:

$$
m(r, \mathbf{a}, S)= \begin{cases}\frac{1}{2 \pi} \int_{0}^{2 \pi} \log ^{+}\left\|\mathbf{x}\left(r e^{i \theta}\right)\right\| d \theta, & \text { for } \mathbf{a}=\infty \\ \frac{1}{2 \pi} \int_{0}^{2 \pi} \log ^{+} \frac{1}{\left\|\mathbf{x}\left(r e^{i \theta}\right)-\mathbf{a}\right\|} d \theta, & \text { for } \mathbf{a} \neq \infty\end{cases}
$$

where $\log ^{+} x=\max (\log x, 0)$ for $x \geq 0$ and $\|\mathbf{x}(z)\|=\sqrt{x_{1}^{2}(z)+x_{2}^{2}(z)+x_{3}^{2}(z)}\left(z=r e^{i \theta}\right)$,

$$
N(r, \mathbf{a}, S)= \begin{cases}\int_{0}^{r} \frac{n(\rho, \infty, S)-n(0, \infty, S)}{\rho} d \rho+n(0, \infty, S) \log r, & \text { for } \mathbf{a}=\infty \\ \int_{0}^{r} \frac{n(\rho, \mathbf{a}, S)-n(0, \mathbf{a}, S)}{\rho} d \rho+n(0, \mathbf{a}, S) \log r, & \text { for } \mathbf{a} \neq \infty,\end{cases}
$$

where $n(r, \mathbf{a}, S)$ and $n(r, \infty, S)$ denote, respectively, the number of a-points $\left(\mathbf{a} \in \mathbb{R}^{3}\right)$ and poles of a meromorphic minimal surface $S$ in the disc $\{z:|z| \leq r\}$, counted according to multiplicity,

$$
H(r, \mathbf{a}, S)= \begin{cases}0, & \text { for } \mathbf{a}=\infty \\ \int_{0}^{r} \frac{h(\rho, \mathbf{a} ; S)}{\rho} d \rho, & \text { for } \mathbf{a} \neq \infty\end{cases}
$$

where

$$
h(\rho, \mathbf{a}, S)=\frac{1}{2 \pi} \iint_{A_{\rho}(0)} \triangle \log \|\mathbf{x}(u, v)-\mathbf{a}\| d u d v, \quad \Delta=\frac{\partial^{2}}{\partial u^{2}}+\frac{\partial^{2}}{\partial v^{2}}
$$

is the Laplace operator and $A_{\rho}(0)$ denotes the closed disc $|z| \leq \rho$. The function $T(r, S)=$ $m(r, \infty, S)+N(r, \infty, S)$ is called the characteristic of a meromorphic minimal surface $S$. The number

$$
\lambda=\varliminf_{r \rightarrow \infty} \frac{\log T(r, S)}{\log r}
$$

is called the lower order of $S$.

In [2] E. F. Beckenbach gets an analog of Nevanlinna's first fundamental theorem for minimal surfaces. The theorem states that if $S$ is a meromorphic minimal surface then for each $\mathbf{a} \in \mathbb{R}^{3}$

$$
m(r, \mathbf{a}, S)+N(r, \mathbf{a}, S)+H(r, \mathbf{a}, S)=T(r, S)+O(1)(r \rightarrow \infty) .
$$

E. F. Beckenbach and T. Cootz in [3] generalize Nenanlinna's second fundamental theorem to minimal surfaces. The theorem says that for a meromorphic minimal surface $S$ and points $\mathbf{a}_{k} \in \mathbb{R}^{3}(k=1, \ldots, q)$ we have the following inequality

$$
\sum_{k=1}^{q} m\left(r, \mathbf{a}_{k}, S\right) \leq 2 T(r, S)+O(\log (r T(r, S))), r \notin E, r \rightarrow \infty,
$$


where $E$ is a set of finite measure. Notice that $N(r, \mathbf{a}, S)$ vanishes almost everywhere in $\mathbb{R}^{3}$ so the most important function in Beckenbach's theory is $H(r, \mathbf{a}, S)$.

2. Main results. In 1979 I. I. Marchenko applied Petrenko's theory of growth of meromorphic functions ([16]) to the theory of meromorphic minimal surfaces. In [13] I. I. Marchenko defined the quantities

$$
\mathcal{L}(r, \mathbf{a}, S)=\left\{\begin{array}{ll}
\max _{|z|=r} \log ^{+} \frac{1}{\|\mathbf{x}(z)-\mathbf{a}\|}, & \text { for } \mathbf{a} \neq \infty ; \\
\max _{|z|=r} \log ^{+}\|\mathbf{x}(z)\|, & \text { for } \mathbf{a}=\infty,
\end{array} \quad \beta(\mathbf{a}, S)=\underline{\lim _{r \rightarrow \infty}} \frac{\mathcal{L}(r, \mathbf{a}, S)}{T(r, S)} .\right.
$$

$\beta(\mathbf{a}, S)$ is called the magnitude of deviation of the meromorphic minimal surface $S$ at the point a. In the paper [13] I. I. Marchenko also obtained a sharp upper estimate of $\beta(\mathbf{a}, S)$ for surfaces of finite lower order.

Theorem ([13]). If $S$ is a meromorphic minimal surface of finite lower order $\lambda$, then for each $\mathbf{a} \in \mathbb{R}^{3}$

$$
\beta(\mathbf{a}, S) \leq \begin{cases}\frac{\pi \lambda}{\sin \pi \lambda}, & \text { for } \lambda \leq \frac{1}{2} \\ \pi \lambda, & \text { for } \lambda>\frac{1}{2}\end{cases}
$$

In 2004 E. Ciechanowicz and I. I. Marchenko applied a quantity measuring the number of separated maximum modulus points of a meromorphic function to obtain an upper estimate of deviation for meromorphic functions ([5], see also [6] and [7]). We define a similar quantity for meromorphic minimal surfaces. Let $\phi(r)$ be a positive, nondecreasing convex function of $\log r$ for $r>0$, such that $\phi(r)=o(T(r, S))$ and $p_{\phi}(r, \infty, S)$ be the number of component intervals of the set $\left\{\theta: \log \left\|\mathbf{x}\left(r e^{i \theta}\right)\right\|>\phi(r)\right\}$ possessing at lest one maximum modulus point of the function $\left\|\mathbf{x}\left(r e^{i \theta}\right)\right\|$. Moreover, let us denote $p_{\phi}(\infty, S)=\liminf _{r \rightarrow \infty} p_{\phi}(r, \infty, S)$. We set

$$
p(\infty, S)=\sup _{\{\phi\}} p_{\phi}(\infty, S) .
$$

It is clear that $p(\infty, S) \geq 1$. In this paper we get an upper estimate of deviation for a meromorphic minimal surface $S$ in terms of $p(\infty, S)$. Our main results are as follows.

Theorem 1. For a meromorphic minimal surface $S$ of finite lower order $\lambda$, we have

$$
\beta(\infty, S) \leq \begin{cases}\frac{\pi \lambda}{p(\infty, S)}, & \text { if } \frac{\lambda}{p(\infty, S)} \geq \frac{1}{2} \\ \frac{\pi \lambda}{\sin \pi \lambda}, & \text { if } p(\infty, S)=1 \text { and } \lambda<\frac{1}{2} \\ \frac{\pi \lambda}{p(\infty, S)} \sin \frac{\pi \lambda}{p(\infty, S)}, & \text { if } p(\infty, S)>1 \text { and } \frac{\lambda}{p(\infty, S)}<\frac{1}{2}\end{cases}
$$

Corollary 1. For a meromorphic minimal surface $S$ of finite lower order $\lambda$, we have

$$
p(\infty, S) \leq \max \left(\left[\frac{\pi \lambda}{\beta(\infty, f)}\right], 1\right),
$$

where $[x]$ is the integer part of $x$.

Corollary 2. For an entire minimal surface $S$ of finite lower order $\lambda$ we have

$$
p(\infty, S) \leq \max ([\pi \lambda], 1)<+\infty .
$$


3. Auxiliary results. Let $S=\left\{x(z)=\left(x_{1}(z), x_{2}(z), x_{3}(z): z \in \mathbb{C}\right\}\right.$ be a meromorphic minimal surface and let $\phi(r)$ be a positive nondecreasing convex function of $\log r$ such that $\phi(r)=o(T(r, S))$. We consider the function given by $u_{\phi}(z)=\max (\log \|\mathbf{x}(z)\|, \phi(|z|))$.

Lemma 1. The function $u_{\phi}(z)$ is a $\delta$-subharmonic function in $\mathbb{C}$.

Proof. Let $\left\{a_{n}\right\}$ be poles of surface $S$. Then, by Weierstrass theorem, we can find an entire function $g(z)$, such that $g\left(a_{n}\right)=0$. We specify a new function $\widetilde{\mathbf{x}}(z)=\left(\widetilde{x_{1}}(z), \widetilde{x_{2}}(z), \widetilde{x_{3}}(z)\right)$ by $\widetilde{\mathbf{x}}(z)=\mathbf{x}(z) \cdot g(z)$, then

$$
\|\widetilde{\mathbf{x}}(z)\|=\|\mathbf{x}(z)\||g(z)| .
$$

We obtain that $\|\mathbf{x}(z)\|=\frac{\|\widetilde{\mathbf{x}}(z)\|}{|g(z)|}$, therefore

$$
\log \|\mathbf{x}(z)\|=\log \frac{\|\widetilde{\mathbf{x}}(z)\|}{|g(z)|}=\log \|\widetilde{\mathbf{x}}(z)\|-\log |g(z)|
$$

By definition of $u_{\phi}(z)$ we have

$$
u_{\phi}(z)=\max (\log \|\widetilde{\mathbf{x}}(z)\|, \log |g(z)|+\phi(|z|))-\log |g(z)| .
$$

The function $\phi(r)$ is a convex function of $\log r$ for $r>0$. Therefore $\phi(|z|)$ is a subharmonic function in $\mathbb{C}$. Also

$$
t(z):=\max (\log \|\widetilde{\mathbf{x}}(z)\|, \log |g(z)|+\phi(|z|))
$$

is subharmonic. Thus $u_{\phi}(z)=t(z)-\log |g(z)|$ is a $\delta$-subharmonic function in $\mathbb{C}$.

Let $([1])$

$$
m^{*}\left(r, \theta, u_{\phi}\right)=\sup _{|E|=2 \theta} \frac{1}{2 \pi} \int_{E} u_{\phi}\left(r e^{i \varphi}\right) d \varphi, T^{*}\left(r, \theta, u_{\phi}\right)=m^{*}\left(r, \theta, u_{\phi}\right)+N(r, \infty, S),
$$

where $r \in(0, \infty), \theta \in[0, \pi], E$ is a measurable set and $|E|$ is the Lebesgue measure of $E$. Now for each $t \in(0,+\infty)$, consider the set $G_{t}=\left\{r e^{i \varphi}: u_{\phi}\left(r e^{i \varphi}\right)>t\right\}$, and let

$$
u_{\phi}^{*}\left(r e^{i \varphi}\right)=\sup \left\{t: r e^{i \varphi} \in G_{t}^{*}\right\} \text {, }
$$

where $G_{t}^{*}$ is the symmetric rearrangement of the set $G_{t}([8])$.

The function $u_{\phi}^{*}\left(r e^{i \varphi}\right)$ is non-negative and non-increasing in the interval $[0, \pi]$, even with respect to $\phi$ and for each fixed $r$ equimeasurable with $u_{\phi}\left(r e^{i \varphi}\right)$. Moreover, it satisfies the equalities

$$
\begin{gathered}
u_{\phi}^{*}(r)=\max \left(\log \max _{|z|=r}\|\mathbf{x}(z)\|, \phi(r)\right), \quad u_{\phi}^{*}\left(r e^{i \pi}\right)=\max \left(\log \min _{|z|=r}\|\mathbf{x}(z)\|, \phi(r)\right), \\
m^{*}\left(r, \theta, u_{\phi}\right)=\frac{1}{\pi} \int_{0}^{\theta} u_{\phi}^{*}\left(r e^{i \varphi}\right) d \varphi .
\end{gathered}
$$

From Baernstein's theorem ([1]), the function $T^{*}\left(r, \theta, u_{\phi}\right)$ is subharmonic in $D=\left\{r e^{i \theta}: 0<r<\infty, 0<\theta<\pi\right\}$, continuous in $D \cup(-\infty, 0) \cup(0, \infty)$ and logarithmically convex in $r>0$ for each fixed $\theta \in[0, \pi]$. Moreover,

$$
T^{*}\left(r, 0, u_{\phi}\right)=N(r, \infty, S), \quad T^{*}\left(r, \pi, u_{\phi}\right)=T(r, S)+o(T(r, S)) \quad(r \rightarrow \infty),
$$




$$
\frac{\partial}{\partial \theta} T^{*}\left(r, \theta, u_{\phi}\right)=\frac{u_{\phi}^{*}\left(r e^{i \theta}\right)}{\pi} \text { for } 0<\theta<\pi .
$$

Let $\alpha(r)$ be a real-valued function of the real variable $r$ and define

$$
L \alpha(r)=\varliminf_{h \rightarrow 0} \frac{\alpha\left(r e^{h}\right)+\alpha\left(r e^{-h}\right)-2 \alpha(r)}{h^{2}} .
$$

When $\alpha(r)$ is twice differentiable in $r$, then $L \alpha(r)=r \frac{d}{d r} r \frac{d}{d r} \alpha(r)$.

Lemma 2. Let $S=\left\{\mathbf{x}(z)=\left(x_{1}(z), x_{2}(z), x_{3}(z)\right): z \in \mathbb{C}\right\}$ be a meromorphic minimal surface. For almost all $\theta \in[0, \pi]$ and for all $r>0$ such that the function $\|\mathbf{x}(z)\|$ has neither zeros nor poles in $\{z:|z|=r\}$, we have

$$
L T^{*}\left(r, \theta, u_{\phi}\right) \geq-\frac{p_{\phi}^{2}(r, \infty, S)}{\pi} \frac{\partial u_{\phi}^{*}(r, \theta)}{\partial \theta}
$$

Proof. In the proof of Lemma 2 we will use the ideas from the proof of Lemma 1 in [14]. Let us assume that $r_{0}$ is a number satisfying the hypothesis. Since $u_{\phi}^{*}\left(r_{0}, \theta\right)$ is a non-increasing function of $\theta$ then by Lebesgue's theorem the derivative $\frac{\partial u_{\phi}^{*}\left(r_{0}, \theta\right)}{\partial \theta}$ exists for almost all $\theta \in[0, \pi]$. Let us choose $\theta \in(0, \pi)$ such that $\frac{\partial u_{\phi}^{*}\left(r_{0}, \theta\right)}{\partial \theta}$ exists.

If $u_{\phi}^{*}\left(r_{0}, \theta\right)=\phi\left(r_{0}\right)$, then $u_{\phi}^{*}\left(r_{0}, x\right)=\phi\left(r_{0}\right)$ for all $x>\theta$, and so $\frac{\partial u_{\phi}^{*}\left(r_{0}, \theta\right)}{\partial \theta}=0$. As $T^{*}\left(r, \theta, u_{\phi}\right)$ is a convex function of $\log r$ we have $L T^{*}\left(r, \theta, u_{\phi}\right) \geq 0$. Therefore the lemma is proved in the case when $\frac{\partial u_{\phi}^{*}\left(r_{0}, \theta\right)}{\partial \theta}=0$ or when $u_{\phi}^{*}\left(r_{0}, \theta\right)=\phi\left(r_{0}\right)$.

Let us assume now that $\frac{\partial u_{\phi}^{*}\left(r_{0}, \theta\right)}{\partial \theta}<0$ and $u_{\phi}^{*}\left(r_{0}, \theta\right)>\phi\left(r_{0}\right)$. There exists a set $E\left(r_{0}, \theta\right)$ ([1]) such that

$$
m^{*}\left(r_{0}, \theta, u_{\phi}\right)=\frac{1}{2 \pi} \int_{E\left(r_{0}, \theta\right)} u_{\phi}\left(r_{0}, \varphi\right) d \varphi
$$

where

$$
\left\{\varphi: u_{\phi}\left(r_{0}, \varphi\right)>u_{\phi}^{*}\left(r_{0}, \theta\right)\right\} \subset E\left(r_{0}, \theta\right) \subset\left\{\varphi: u_{\phi}\left(r_{0}, \varphi\right) \geq u_{\phi}^{*}\left(r_{0}, \theta\right)\right\} .
$$

Let us now consider the function $F(\varphi)=\log \left\|\mathbf{x}\left(r_{0} e^{i \varphi}\right)\right\|$. Then the set $\left\{\varphi: F(\varphi)=u_{\phi}^{*}\left(r_{0}, \theta\right)\right\}$ is finite. Otherwise there would exist a convergent sequence $\left(\varphi_{k}\right)$ such that $F\left(\varphi_{k}\right)=u_{\phi}^{*}\left(r_{0}, \theta\right)$. As $r_{0}$ is chosen so that there are neither zeros nor poles of $\left\|\mathbf{x}\left(r_{0} e^{i \varphi}\right)\right\|$ on the circle $|z|=r_{0}$, the function $F(\varphi)$ is an analytic function of $\varphi \in[0,2 \pi]$. Applying the uniqueness theorem, we can state that if $F\left(\varphi_{k}\right)=u_{\phi}^{*}\left(r_{0}, \theta\right)$ then $F(\varphi)=u_{\phi}^{*}\left(r_{0}, \theta\right)$ for all $\varphi \in[0,2 \pi]$. This means that $u_{\phi}\left(r_{0}, \varphi\right)=u_{\phi}^{*}\left(r_{0}, \theta\right)$ for all $\varphi \in[0,2 \pi]$ and as a result $\frac{\partial u_{\phi}^{*}\left(r_{0}, \theta\right)}{\partial \theta}=0$, which is a contradiction. Therefore the set $\left\{\varphi: F(\varphi)=u_{\phi}^{*}\left(r_{0}, \theta\right)\right\}$ is indeed finite. As a result we have

$$
m^{*}\left(r_{0}, \theta, u_{\phi}\right)=\frac{1}{2 \pi} \int_{E_{1}\left(r_{0}, \theta\right)} u_{\phi}\left(r_{0}, \varphi\right) d \varphi
$$

where $E_{1}\left(r_{0}, \theta\right)=\left\{\varphi: u_{\phi}\left(r_{0}, \varphi\right)>u_{\phi}^{*}\left(r_{0}, \theta\right)\right\}$.

Let us now consider for $r>0$ the function

$$
\Psi(r)=\frac{1}{2 \pi} \int_{E_{1}\left(r_{0}, \theta\right)} u_{\phi}(r, \varphi) d \varphi .
$$


We have $\Psi\left(r_{0}\right)=m^{*}\left(r_{0}, \theta, u_{\phi}\right)$ and $\Psi(r) \leq m^{*}\left(r, \theta, u_{\phi}\right)$ for all $r>0$. Hence $L m^{*}\left(r_{0}, \theta, u_{\phi}\right)$ $\geq L \Psi\left(r_{0}\right)$. Since the set $E_{1}\left(r_{0}, \theta\right)$ is an open subset of the circle $|z|=r_{0}$, it implies that $E_{1}\left(r_{0}, \theta\right)=\bigcup_{k}\left(\alpha_{k}, \beta_{k}\right)$. The function $F(\varphi)$ is analytic for all $\varphi \in[0,2 \pi]$ therefore $F\left(\alpha_{k}\right)=$ $F\left(\beta_{k}\right)=u_{\phi}^{*}\left(r_{0}, \theta\right)$ and it follows again from the uniqueness theorem that the family of intervals $\left(\alpha_{k}, \beta_{k}\right)$ is finite. Let $m=m\left(r_{0}\right)$ denote the number of those intervals.

In [2] E. F. Beckenbach obtained

$$
\Delta \log \left\|\mathbf{x}\left(r e^{i \varphi}\right)\right\|=\frac{2\left(\mathbf{x}\left(r e^{i \varphi}\right), \mathbf{X}\left(r e^{i \varphi}\right)\right)^{2} \eta}{\left\|\mathbf{x}\left(r e^{i \varphi}\right)\right\|^{2}} \geq 0,
$$

where $\mathbf{X}\left(r e^{i \varphi}\right)$ is the unit normal vector for $S$ and

$$
\eta=\sum_{j=1}^{3}\left(\frac{\partial x_{j}}{\partial u}\right)^{2}=\sum_{j=1}^{3}\left(\frac{\partial x_{j}}{\partial v}\right)^{2}
$$

denotes the equal coefficients of the first fundamental form. It follows that the function $\log \|\mathbf{x}(z)\|$ is subharmonic on a certain neighborhood of the circle $|z|=r_{0}$, as $S$ has neither zeros nor poles on this circle. Therefore

$$
\begin{gathered}
L \Psi\left(r_{0}\right)=\left.\frac{1}{2 \pi} \sum_{k=1}^{m} \int_{\alpha_{k}}^{\beta_{k}} r \frac{d}{d r} r \frac{d}{d r} u_{\phi}\left(r e^{i \varphi}\right)\right|_{r=r_{0}} d \varphi= \\
=\left.\frac{1}{2 \pi} \sum_{k=1}^{m} \int_{\alpha_{k}}^{\beta_{k}} r \frac{d}{d r} r \frac{d}{d r} \log \left\|\mathbf{x}\left(r e^{i \varphi}\right)\right\|\right|_{r=r_{0}} d \varphi= \\
=\left.\frac{1}{2 \pi} \sum_{k=1}^{m} \int_{\alpha_{k}}^{\beta_{k}}\left(r^{2} \Delta \log \left\|\mathbf{x}\left(r e^{i \varphi}\right)\right\|-\frac{\partial^{2} \log \left\|\mathbf{x}\left(r e^{i \varphi}\right)\right\|}{\partial \varphi^{2}}\right)\right|_{r=r_{0}} d \varphi \geq-\left.\frac{1}{2 \pi} \sum_{k=1}^{m}\left[\frac{\partial u_{\phi}\left(r_{0}, \varphi\right)}{\partial \varphi}\right]\right|_{\alpha_{k}} ^{\beta_{k}} .
\end{gathered}
$$

Finally, it follows from our previous considerations that

$$
L m^{*}\left(r_{0}, \theta, u_{\phi}\right) \geq L \Psi\left(r_{0}\right) \geq-\left.\frac{1}{2 \pi} \sum_{k=1}^{m}\left[\frac{\partial u_{\phi}\left(r_{0}, \varphi\right)}{\partial \varphi}\right]\right|_{\alpha_{k}} ^{\beta_{k}} .
$$

Now we prove that

$$
L m^{*}\left(r_{0}, \theta, u_{\phi}\right) \geq-\frac{m}{\pi} \frac{\partial u_{\phi}^{*}(r, \theta)}{\partial \theta} .
$$

Note that there exist neighborhoods of points $\alpha_{k}, \beta_{k}(k=1,2, \ldots, m)$ in which the function $F(\varphi)=\log \left\|\mathbf{x}\left(r e^{i \varphi}\right)\right\|$ is, accordingly, strictly increasing or strictly decreasing. If not, in a neighborhood of one of the numbers $\alpha_{k}, \beta_{k}$ there would exist a convergent sequence $\varphi_{k}$ tending to this number and such $F^{\prime}\left(\varphi_{k}\right)=0$. The function $F^{\prime}(\varphi)$ is analytic for $\varphi \in[0,2 \pi]$ and applying the uniqueness theorem, we can state that $F^{\prime}(\varphi)=0$ for all $\varphi \in[0,2 \pi]$. Then for all $\varphi \in[0,2 \pi], \quad F(\varphi)=\log \left\|\mathbf{x}\left(r_{0} e^{i \varphi}\right)\right\|=u_{\phi}^{*}\left(r_{0}, \theta\right)$, therefore $\frac{\partial u_{\phi}^{*}\left(r_{0}, \theta\right)}{\partial \theta}=0$, which is a contradiction as we assumed that $\frac{\partial u_{\phi}^{*}\left(r_{0}, \theta\right)}{\partial \theta}<0$.

This leads us to the conclusion that in some neighborhoods of points $\alpha_{k}, \beta_{k}(k=$ $1,2 \ldots, m)$ the function $\log \left\|\mathbf{x}\left(r_{0} e^{i \varphi}\right)\right\|$ is strictly monotone.

Since $u_{\phi}^{*}\left(r_{0}, \theta\right)>0$ by definition of the function $\log ^{+}(x)$ there exist neighborhoods of the points $\alpha_{k}, \beta_{k}$ where $u_{\phi}\left(r_{0}, \theta\right)=\log \left\|\mathbf{x}\left(r_{0} e^{i \varphi}\right)\right\|$. It follows from the uniqueness theorem that $u_{\phi}\left(r_{0}, \theta\right)$ is strictly monotone in the neighborhood of all points $\alpha_{k}, \beta_{k}(k=1,2, \ldots, m)$. 
We want to show that $\frac{\partial u_{\phi}\left(r_{0}, \alpha_{k}\right)}{\partial \varphi}>0, \frac{\partial u_{\phi}\left(r_{0}, \beta_{k}\right)}{\partial \varphi}<0$ for all $k=1,2, \ldots, m$. Let us choose $h>0$, such that $u_{\phi}\left(r_{0}, \varphi\right)$ is strictly increasing in the $h$-neighborhood of the point $\alpha_{k}$. Then we have

$$
\mu\left\{\varphi: u_{\phi}\left(r_{0}, \varphi\right) \geq u_{\phi}\left(r_{0}, \alpha_{k}+h\right)\right\} \leq 2 \theta-h,
$$

where $\mu$ is the Lebesgue measure. The function $u_{\phi}(r, \theta)$ is equimeasurable with $u_{\phi}^{*}\left(r_{0}, \theta\right)$ so we obtain

$$
\mu\left\{\varphi: u_{\phi}\left(r_{0}, \varphi\right) \geq u_{\phi}^{*}\left(r_{0}, \theta-\frac{h}{2}\right)\right\}=\mu\left\{\varphi: u_{\phi}^{*}\left(r_{0}, \varphi\right) \geq u_{\phi}^{*}\left(r_{0}, \theta-\frac{h}{2}\right)\right\}=2 \theta-h .
$$

This, together with (3) allows us to write $u_{\phi}\left(r_{0}, \alpha_{k}+h\right) \geq u_{\phi}^{*}\left(r_{0}, \theta-\frac{h}{2}\right)$. Therefore

$$
u_{\phi}\left(r_{0}, \alpha_{k}\right)=u_{\phi}^{*}\left(r_{0}, \theta\right) \text {, }
$$

and for $h>0$ we have

$$
\frac{u_{\phi}\left(r_{0}, \alpha_{k}+h\right)-u_{\phi}\left(r_{0}, \alpha_{k}\right)}{h} \geq \frac{u_{\phi}^{*}\left(r_{0}, \theta-\frac{h}{2}\right)-u_{\phi}^{*}\left(r_{0}, \theta\right)}{h} .
$$

Taking the limit of both sides with $h \rightarrow 0^{+}$we have

$$
\left.\frac{\partial u_{\phi}\left(r_{0}, \varphi\right)}{\partial \varphi}\right|_{\alpha_{k}} \geq-\frac{1}{2} \frac{\partial u_{\phi}^{*}\left(r_{0}, \theta\right)}{\partial \theta}, \quad(k=1,2, \ldots, m) .
$$

From our assumption $\frac{\partial u_{\phi}\left(r_{0}, \theta\right)}{\partial \theta}<0$, hence

$$
\frac{\partial u_{\phi}\left(r_{0}, \alpha_{k}\right)}{\partial \varphi} \geq-\frac{1}{2} \frac{\partial u_{\phi}\left(r_{0}, \theta\right)}{\partial \theta}>0, \quad(k=1,2, \ldots, m)
$$

Similarly we show that $\frac{\partial u_{\phi}\left(r_{0}, \beta_{k}\right)}{\partial \varphi}<0$. Let us choose $h>0$ such that $u_{\phi}\left(r_{0}, \varphi\right)$ is a strictly increasing function in the $h$-neighborhood of the point $\beta_{k}$. Then we have

$$
\mu\left\{\varphi: u_{\phi}\left(r_{0}, \varphi\right) \geq u_{\phi}\left(r_{0}, \beta_{k}-h\right)\right\} \leq 2 \theta-h .
$$

The function $u_{\phi}\left(r_{0}, \theta\right)$ is equimeasurable with $u_{\phi}^{*}\left(r_{0}, \theta\right)$ so by the inequalities (4) i (6) we obtain $u_{\phi}\left(r_{0}, \beta_{k}-h\right) \geq u_{\phi}^{*}\left(r_{0}, \theta-\frac{h}{2}\right)$.

Hence

$$
u_{\phi}\left(r_{0}, \beta_{k}\right) \geq u_{\phi}^{*}\left(r_{0}, \theta\right),
$$

and for $h>0$

$$
\frac{u_{\phi}\left(r_{0}, \beta_{k}-h\right)-u_{\phi}\left(r_{0}, \beta_{k}\right)}{h} \geq \frac{u_{\phi}^{*}\left(r_{0}, \theta-\frac{h}{2}\right)-u_{\phi}^{*}\left(r_{0}, \theta\right)}{h} .
$$

If $h \rightarrow 0^{+}$we have

$$
-\left.\frac{\partial u_{\phi}\left(r_{0}, \varphi\right)}{\partial \varphi}\right|_{\beta_{k}} \geq-\frac{1}{2} \frac{\partial u_{\phi}^{*}\left(r_{0}, \theta\right)}{\partial \theta} .
$$

Since we assumed that $\frac{\partial u_{\phi}^{*}\left(r_{0}, \theta\right)}{\partial \theta}<0$, we obtain

$$
\left.\frac{\partial u_{\phi}\left(r_{0}, \varphi\right)}{\partial \varphi}\right|_{\beta_{k}} \leq \frac{1}{2} \frac{\partial u_{\phi}^{*}\left(r_{0}, \theta\right)}{\partial \theta}<0 .
$$


We showed that

$$
\frac{\partial u_{\phi}\left(r_{0}, \alpha_{k}\right)}{\partial \varphi}>0, \quad \frac{\partial u_{\phi}\left(r_{0}, \beta_{k}\right)}{\partial \varphi}<0, \quad \text { for } k=1,2, \ldots, m
$$

Let $h_{0}>0$ be a positive number such that for all $\varphi$, satisfying the condition $|\varphi| \leq h_{0}$, we have the inequalities

$$
\alpha_{k}+h_{0}<\beta_{k}-h_{0}, \frac{\partial u_{\phi}\left(r_{0}, \alpha_{k}+\varphi\right)}{\partial \varphi}>0 \text { and } \frac{\partial u_{\phi}\left(r_{0}, \beta_{k}+\varphi\right)}{\partial \varphi}<0 \text { for } k=1,2, \ldots, m \text {. }
$$

Let us denote by $\gamma_{k}$ the lowest value of $u_{\phi}\left(r_{0}, \varphi\right)$ on the interval $\left[\alpha_{k}+h_{0}, \beta_{k}-h_{0}\right]$. Let also $\gamma=\min _{1 \leq k \leq m} \gamma_{k}$. Then from (5) we have $u_{\phi}\left(r_{0}, \alpha_{1}+h_{0}\right) \geq \gamma>u_{\phi}\left(r_{0}, h_{1}\right)=u_{\phi}^{*}\left(r_{0}, \theta\right)$.

Now we choose the number $h_{1}$ such that $0<h_{1} \leq h_{0}$ and $u_{\phi}\left(r_{0}, \alpha_{1}+h_{1}\right)=\gamma$. From the choice of $h_{1}$ the equations

$$
\left\{\begin{array}{l}
u_{\phi}\left(r_{0}, \beta_{k}-x\right)=u_{\phi}\left(r_{0}, \alpha_{1}+h\right), \\
u_{\phi}\left(r_{0}, \alpha_{k}+y\right)=u_{\phi}\left(r_{0}, \alpha_{1}+h\right),
\end{array}\right.
$$

have always just one pair of solutions for all $0<h<h_{1}$. Let us denote these solutions by $x_{k}(h)$ and $y_{k}(h)$. From the continuity of the function $u_{\phi}\left(r_{0}, \varphi\right)$ and the equality $u_{\phi}\left(r_{0}, \beta_{k}\right)=$ $u_{\phi}\left(r_{0}, \alpha_{k}\right)=u_{\phi}^{*}\left(r_{0}, \theta\right) \quad(k=1,2, \ldots, m)$ we obtain that $x_{k}(h) \rightarrow 0, y_{k}(h) \rightarrow 0$ when $h \rightarrow 0$, because $u_{\phi}\left(r_{0}, \beta_{k}-x_{k}(h)\right)=u_{\phi}\left(r_{0}, \alpha_{k}\right)=u_{\phi}\left(\alpha_{1}+h\right) \rightarrow u_{\phi}^{*}\left(r_{0}, \theta\right)\left(h \rightarrow 0^{+}\right)$.

On the other hand, $u_{\phi}\left(r_{0}, \beta_{k}\right)=u_{\phi}^{*}\left(r_{0}, \theta\right)$, hence $x_{k}(h) \rightarrow 0$ when $h \rightarrow 0^{+}$. We show now that by the differentiability of the function $u_{\phi}\left(r_{0}, \varphi\right)$, we get the inequality

$$
u_{\phi}\left(r_{0}, \beta_{k}\right)-u_{\phi}^{\prime}\left(r_{0}, \beta_{k}\right) \cdot x_{k}+o\left(x_{k}\right)=u_{\phi}\left(r_{0}, \alpha_{1}\right)+u_{\phi}^{\prime}\left(r_{0}, \alpha_{1}\right) \cdot h+o(h)(h \rightarrow 0),
$$

where by $u_{\phi}^{\prime}\left(r_{0}, \beta_{k}\right)$ we mean $\frac{\partial u_{\phi}\left(r_{0}, \varphi\right)}{\partial \varphi}$. By definition we have

$$
u_{\phi}^{\prime}\left(r_{0}, \beta_{k}\right)=\lim _{x_{k} \rightarrow 0} \frac{u_{\phi}\left(r_{0}, \beta_{k}-x_{k}\right)-u_{\phi}\left(r_{0}, \beta_{k}\right)}{-x_{k}},
$$

therefore

$$
u_{\phi}^{\prime}\left(r_{0}, \beta_{k}\right)=\frac{u_{\phi}\left(r_{0}, \beta_{k}-x_{k}\right)-u_{\phi}\left(r_{0}, \beta_{k}\right)}{-x_{k}}+o(1)\left(x_{k} \rightarrow 0\right) .
$$

Hence $\left(-x_{k}\right) \cdot u_{\phi}^{\prime}\left(r_{0}, \beta_{k}\right)=u_{\phi}\left(r_{0}, \beta_{k}-x_{k}\right)-u_{\phi}\left(r_{0}, \beta_{k}\right)+o\left(x_{k}\right)$, so we have $u_{\phi}\left(r_{0}, \beta_{k}\right)-$ $x_{k} \cdot u_{\phi}^{\prime}\left(r_{0}, \beta_{k}\right)+o\left(x_{k}\right)=u_{\phi}\left(r_{0}, \beta_{k}-x_{k}\right)$.

On the other hand, $u_{\phi}\left(r_{0}, \beta_{k}-x_{k}\right)=u_{\phi}\left(r_{0}, \alpha_{1}+h\right)$, and we obtain

$$
u_{\phi}\left(r_{0}, \alpha_{1}+h\right)=u_{\phi}\left(r_{0}, \alpha_{1}\right)+u_{\phi}^{\prime}\left(r_{0}, \alpha_{1}\right) \cdot h+o(h) .
$$

From the equality proved above it follows that

$$
x_{k}=-\frac{u_{\phi}^{\prime}\left(r_{0}, \alpha_{1}\right)}{u_{\phi}^{\prime}\left(r_{0}, \beta_{k}\right)} \cdot h+o(h), \quad h \rightarrow 0 \quad(k=1,2, \ldots, m) .
$$

Performing similar operations we can show that

$$
y_{k}=\frac{u_{\phi}^{\prime}\left(r_{0}, \alpha_{1}\right)}{u_{\phi}^{\prime}\left(r_{0}, \alpha_{k}\right)} \cdot h+o(h), \quad h \rightarrow 0 \quad(k=1,2, \ldots, m) .
$$


However, from the choice of $x_{k}, y_{k}$ we have

$$
\begin{gathered}
\mu\left\{\varphi: u_{\phi}\left(r_{0}, \varphi\right) \geq u_{\phi}\left(r_{0}, \alpha_{1}+h\right)\right\}=2 \theta-\sum_{k=1}^{m}\left(x_{k}+y_{k}\right)= \\
=2 \theta-\sum_{k=1}^{m}\left(\frac{u_{\phi}^{\prime}\left(r_{0}, \alpha_{1}\right)}{u_{\phi}^{\prime}\left(r_{0}, \alpha_{k}\right)}-\frac{u_{\phi}^{\prime}\left(r_{0}, \alpha_{1}\right)}{u_{\phi}^{\prime}\left(r_{0}, \beta_{k}\right)}\right) h+o(h)=2 \theta-A(h),
\end{gathered}
$$

where

$$
A(h)=\sum_{k=1}^{m}\left(\frac{u_{\phi}^{\prime}\left(r_{0}, \alpha_{1}\right)}{u_{\phi}^{\prime}\left(r_{0}, \alpha_{k}\right)}-\frac{u_{\phi}^{\prime}\left(r_{0}, \alpha_{1}\right)}{u_{\phi}^{\prime}\left(r_{0}, \beta_{k}\right)}\right) h+o(h) .
$$

But $\mu\left\{\varphi: u_{\phi}^{*}\left(r_{0}, \varphi\right) \geq u_{\phi}^{*}\left(r_{0}, \theta-\frac{1}{2} A(h)\right)\right\}=2 \theta-A(h)$, so we have $u_{\phi}^{*}\left(r_{0}, \theta-\frac{1}{2} A(h)\right)=$ $u_{\phi}\left(r_{0}, \alpha_{1}+h\right)$. The function $u_{\phi}^{*}\left(r_{0}, \varphi\right)$ is differentiable at the point $\theta$, hence

$$
u_{\phi}^{*}\left(r_{0}, \theta\right)-\frac{1}{2}\left(u_{\phi}^{*}\left(r_{0}, \theta\right)\right)^{\prime} A(h)+o(A(h))=u_{\phi}\left(r_{0}, \alpha_{1}\right)+u_{\phi}^{\prime}\left(r_{0}, \alpha_{1}\right) \cdot h+o(h) \quad(h \rightarrow 0) .
$$

Since $u_{\phi}\left(r_{0}, \alpha_{1}\right)=u_{\phi}^{*}\left(r_{0}, \theta\right)$ we obtain

$$
-\frac{1}{2}\left(u_{\phi}^{*}\left(r_{0}, \theta\right)\right)^{\prime} \sum_{k=1}^{m}\left(\frac{1}{u_{\phi}^{\prime}\left(r_{0}, \alpha_{k}\right)}-\frac{1}{u_{\phi}^{\prime}\left(r_{0}, \beta_{k}\right)}\right) \cdot u_{\phi}^{\prime}\left(r_{0}, \alpha_{1}\right) \cdot h=u_{\phi}^{\prime}\left(r_{0}, \alpha_{1}\right) \cdot h+o(h) \quad(h \rightarrow 0) .
$$

Since $u_{\phi}^{\prime}\left(r_{0}, \alpha_{1}\right)>0$, multiplying both sides of the equality above by $u_{\phi}^{\prime}\left(r_{0}, \alpha_{1}\right) \cdot h$, we get

$$
1=-\frac{1}{2}\left(u_{\phi}^{*}\left(r_{0}, \theta\right)\right)^{\prime} \sum_{k=1}^{m}\left(\frac{1}{u_{\phi}^{\prime}\left(r_{0}, \alpha_{k}\right)}-\frac{1}{u_{\phi}^{\prime}\left(r_{0}, \beta_{k}\right)}\right) .
$$

Multiplying both sides of this equality by $\sum_{i=1}^{m}\left(u_{\phi}^{\prime}\left(r_{0}, \alpha_{i}\right)-u_{\phi}^{\prime}\left(r_{0}, \beta_{i}\right)\right)$ we have

$$
\begin{gathered}
\sum_{i=1}^{m}\left(u_{\phi}^{\prime}\left(r_{0}, \alpha_{i}\right)-u_{\phi}^{\prime}\left(r_{0}, \beta_{i}\right)\right)= \\
=-\frac{1}{2}\left(u_{\phi}^{*}\left(r_{0}, \theta\right)\right)^{\prime} \sum_{k, i=1}^{m}\left(u_{\phi}^{\prime}\left(r_{0}, \alpha_{i}\right)-u_{\phi}^{\prime}\left(r_{0}, \beta_{i}\right)\right)\left(\frac{1}{u_{\phi}^{\prime}\left(r_{0}, \alpha_{k}\right)}-\frac{1}{u_{\phi}^{\prime}\left(r_{0}, \beta_{k}\right)}\right) .
\end{gathered}
$$

It is easy to show by induction that for each positive numbers $a_{k}, b_{k} \quad(k=1,2, \ldots, m)$ we have

$$
\sum_{k, i=1}^{m}\left(a_{i}+b_{i}\right)\left(\frac{1}{a_{k}}-\frac{1}{b_{k}}\right) \geq 4 m^{2} .
$$

Applying this inequality to the right side of the equality (8) we obtain

$$
\sum_{i=1}^{m}\left(u_{\phi}^{\prime}\left(r_{0}, \alpha_{i}\right)-u_{\phi}^{\prime}\left(r_{0}, \beta_{i}\right)\right) \geq-\frac{1}{2}\left(u_{\phi}^{*}\left(r_{0}, \theta\right)\right)^{\prime} 4 m^{2}=-2 m^{2}\left(u_{\phi}^{*}\left(r_{0}, \theta\right)\right)^{\prime} .
$$

Hence from (2) and (10) we have $L m^{*}\left(r_{0}, \theta, u_{\phi}\right) \geq-\frac{m^{2}}{\pi}\left(u_{\phi}^{*}\left(r_{0}, \theta\right)\right)^{\prime}$. By definition, $p_{\phi}\left(r_{0}, \infty, S\right)$ is the number of component intervals of the set $\left\{\theta:\left\|\mathbf{x}\left(r_{0} e^{i \theta}\right)\right\|>\phi\left(r_{0}\right)\right\}$ possessing at least one 
maximum modulus point of $\left\|\mathbf{x}\left(r_{0} e^{i \theta}\right)\right\|$. On the other hand, $m_{0}$ is the number of component intervals of the set $E_{1}\left(r_{0}, \theta\right)=\left\{\varphi: u_{\phi}\left(r_{0}, \varphi\right)>u^{*}\left(r_{0}, \theta\right)\right\}$ and $u^{*}\left(r_{0}, \theta\right) \geq \phi\left(r_{0}\right)$. Therefore $m \geq p_{\phi}\left(r_{0}, \infty, S\right)$. Also $L T^{*}\left(r_{0}, \theta, u_{\phi}\right) \geq L m^{*}\left(r_{0}, \theta, u_{\phi}\right)$, so we finally receive

$$
L T^{*}\left(r, \theta, u_{\phi}\right) \geq-\frac{p_{\phi}^{2}(r, \infty, S)}{\pi} \frac{\partial u_{\phi}^{*}(r, \theta)}{\partial \theta}
$$

In order to proceed we need the following two lemmas.

Lemma 3 ([14]). If $f(x)$ is a non-decreasing function on $[a, b]$ and $g(x)$ is a non-negative function with the first derivative bounded on $[a, b]$, then

$$
\int_{a}^{b} f^{\prime}(x) g(x) d x \leq f(b) g(b)-f(a) g(a)-\int_{a}^{b} g^{\prime}(x) f(x) d x
$$

Lemma 4 ([16]). Let $S$ be a meromorphic minimal surface of lower order $\lambda$. Then for each $\varepsilon>0$ there exist sequences $S_{k}, R_{k}$ tending to infinity, such that $\lim _{k \rightarrow \infty} \frac{S_{k}}{R_{k}}=0$ and for all $k \geq k_{0}(\varepsilon)$,

$$
\frac{T\left(2 R_{k}, S\right)}{R_{k}^{\lambda}}+\frac{T\left(2 S_{k}, S\right)}{S_{k}^{\lambda}}<\varepsilon \int_{2 S_{k}}^{R_{k}} \frac{T(r, S)}{r^{\lambda+1}} d r .
$$

\section{The upper estimate of the magnitude of deviation.}

Proof. If $\beta(\infty, S)=0$, then the theorem is obviously fulfilled.

Let us assume that $\beta(\infty, S)>0$. Then for every $\phi$ we have $p_{\phi}(\infty, S) \geq 1$.

Let us consider the case of $\lambda>0$ and $p_{\phi}(\infty, S)<\infty$. Now we choose numbers $\alpha$ and $\psi$ satisfying the inequalities

$$
0<\alpha \leq \min \left(\pi, \frac{\pi p_{\phi}(\infty, S)}{2 \lambda}-\alpha\right), \quad-\frac{\pi p_{\phi}(\infty, S)}{2 \lambda} \leq \psi \leq \frac{\pi p_{\phi}(\infty, S)}{2 \lambda}-\alpha .
$$

Moreover, we put ([9] and [10])

$$
\sigma(r)=\int_{0}^{\alpha} T^{*}\left(r, \varphi, u_{\phi}\right) \cos \frac{\lambda(\varphi+\psi)}{p_{\phi}(\infty, S)} d \varphi
$$

Applying the Fatou lemma, we obtain

$$
L \sigma(r)=L \int_{0}^{\alpha} T^{*}\left(r, \varphi, u_{\phi}\right) \cos \frac{\lambda(\varphi+\psi)}{p_{\phi}(\infty, S)} d \varphi \geq \int_{0}^{\alpha} L T^{*}\left(r, \varphi, u_{\phi}\right) \cos \frac{\lambda(\varphi+\psi)}{p_{\phi}(\infty, S)} d \varphi \geq 0 .
$$

It follows from this inequality that $\sigma(r)$ is a convex function of $\log r$, and so $\sigma_{-}^{\prime}(r)$ (the left derivative of $\sigma(r)$ at the point $r)$ is an increasing function in $(0, \infty)$. Therefore, for almost all $r>0, L \sigma(r)=r \frac{d}{d r} r \sigma_{-}^{\prime}(r)$. From the inequality (11) and Lemma 1 it follows that for almost all $r>0$,

$$
L \sigma(r)=r \frac{d}{d r} r \sigma_{-}^{\prime}(r) \geq-\int_{0}^{\alpha} \frac{p_{\phi}^{2}(r, \infty, S)}{\pi} \frac{\partial u_{\phi}^{*}(r, \theta)}{\partial \theta} \cos \frac{\lambda(\varphi+\psi)}{p_{\phi}(\infty, S)} d \theta .
$$


By definition, $p_{\phi}(r, \infty, S)$ assumes only integral values. Thus for $r \geq r_{0}$, there is $p_{\phi}(\infty, S) \leq p_{\phi}(r, \infty, S)$. From this and from (12) it follows that for almost all $r \geq r_{0}$,

$$
L \sigma(r)=r \frac{d}{d r} r \sigma_{-}^{\prime}(r) \geq-\int_{0}^{\alpha} \frac{p_{\phi}^{2}(\infty, S)}{\pi} \frac{\partial u_{\phi}^{*}(r, \theta)}{\partial \theta} \cos \frac{\lambda(\varphi+\psi)}{p_{\phi}(\infty, S)} d \theta .
$$

If there are neither zeros nor poles of $\|\mathbf{x}(z)\|$ on the circle $|z|=r$ for $r>0$, the function $u_{\phi}(r, \theta)$ fulfills the Lipschitz condition on $[0, \pi]$. Therefore $u_{\phi}^{*}(r, \theta)$ also fulfills the Lipschitz condition on $[0, \pi]$. It implies that the function $u_{\phi}^{*}(r, \theta)$ is absolutely continuous on $[0, \pi]$ ([12]). Integrating the equality (13) twice by parts, using properties of functions $T^{*}(r, \theta)$ and $u_{\phi}^{*}(r, 0)$, and by the definition of $\sigma(r)$ we get that for almost all $r \geq r_{0}$

$$
r \frac{d}{d r} r \sigma_{-}^{\prime}(r) \geq h(r)+\lambda^{2} \sigma(r)
$$

where

$$
\begin{gathered}
h(r):=-\frac{p_{\phi}^{2}(\infty, S)}{\pi} u_{\phi}^{*}(r, \alpha) \cos \frac{\lambda(\alpha+\psi)}{p_{\phi}(\infty, S)}+ \\
+\frac{p_{\phi}^{2}(\infty, S)}{\pi}\left(\max \left(\log \max _{|z|=r}\|\mathbf{x}(z)\|, \phi(r)\right) \cos \frac{\lambda \psi}{p_{\phi}(\infty, S)}-\right. \\
\left.-\frac{\lambda \pi}{p_{\phi}(\infty, S)} T^{*}(r, \alpha) \sin \frac{\lambda(\alpha+\psi)}{p_{\phi}(\infty, S)}\right)+\lambda p_{\phi}(\infty, S) N(r, \infty, S) \sin \frac{\lambda \psi}{p_{\phi}(\infty, S)}
\end{gathered}
$$

Dividing both sides by $r^{\lambda+1}$ and integrating by parts over the interval $\left[2 S_{k}, R_{k}\right]$, where $S_{k}, R_{k}$ are the sequences described in Lemma 4, we have

$$
\int_{2 S_{k}}^{R_{k}} \frac{h(r)}{r^{\lambda+1}} d r+\lambda^{2} \int_{2 S_{k}}^{R_{k}} \frac{\sigma(r)}{r^{\lambda+1}} d r \leq \int_{2 S_{k}}^{R_{k}} \frac{1}{r^{\lambda}} \frac{d}{d r} r \sigma_{-}^{\prime}(r) d r=I .
$$

Applying Lemma 4 we get

$$
I \leq\left.\frac{\sigma_{-}^{\prime}(r)}{r^{\lambda+1}}\right|_{2 S_{k}} ^{R_{k}}+\lambda \int_{2 S_{k}}^{R_{k}} \frac{\sigma_{-}^{\prime}(r)}{r^{\lambda}} d r .
$$

Since the function $\sigma(r)$ is a convex function of $\log r$ on the interval $(0,+\infty)$ it follows that the function $f(t)=\sigma\left(e^{t}\right)$ fulfills a Lipschitz condition on each interval $[a, b] \subset(0,+\infty)$, and so the function $\sigma(r)$ is absolutely continuous in these intervals. Integrating by parts the integral in the inequality (16) we have

$$
\int_{2 S_{k}}^{R_{k}} \frac{\sigma_{-}^{\prime}(r)}{r^{\lambda}} d r=\int_{2 S_{k}}^{R_{k}} \frac{\sigma^{\prime}(r)}{r^{\lambda}} d r=\frac{\sigma\left(R_{k}\right)}{R_{k}^{\lambda}}-\frac{\sigma\left(2 S_{k}\right)}{\left(2 S_{k}\right)^{\lambda}}+\lambda \int_{2 S_{k}}^{R_{k}} \frac{\sigma(r)}{r^{\lambda+1}} d r .
$$

Therefore from (15) and (17) we obtain

$$
\int_{2 S_{k}}^{R_{k}} \frac{h(r)}{r^{\lambda+1}} d r \leq\left.\left(\frac{\sigma_{-}^{\prime}(r)}{r^{\lambda-1}}+\lambda \frac{\sigma(r)}{r^{\lambda}}\right)\right|_{2 S_{k}} ^{R_{k}} .
$$

By the definition of $\sigma(r)$, taking any $R \geq 0$ we get

$$
0 \leq \sigma(R) \leq \pi T(R, S) .
$$


The function $r \sigma_{-}^{\prime}(r)$ is non-decreasing on $(0, \infty)$, hence $\sigma(2 R) \geq \sigma(2 R)-\sigma(R)=\int_{R}^{2 R} \sigma^{\prime}(r) d r=\int_{R}^{2 R} \frac{r \sigma_{-}^{\prime}(r)}{r} d r \geq R \sigma_{-}^{\prime}(R) \int_{R}^{2 R} \frac{d r}{r}=R \sigma_{-}^{\prime}(R) \log 2$.

From the observations above, for $R>0$ we have the following inequalities

$$
R \sigma_{-}^{\prime}(R) \leq \frac{1}{\log 2} \sigma(2 R) \leq \frac{\pi}{\log 2} T(2 R, S)
$$

and for $R \geq 1$, by the monotonicity of the function $R \sigma_{-}^{\prime}(R)$, we have

$$
R \sigma_{-}^{\prime}(2 R) \geq \sigma_{-}^{\prime}(2) \text {. }
$$

From (17)-(20) we see that the following inequality holds

$$
\begin{aligned}
\int_{2 S_{k}}^{R_{k}} \frac{h(r)}{r^{\lambda+1}} d r & \leq \frac{\sigma_{-}^{\prime}\left(R_{k}\right)}{R_{k}^{\lambda}}+\lambda \frac{\sigma\left(R_{k}\right)}{R_{k}^{\lambda}}-\frac{\sigma_{-}^{\prime}\left(2 S_{k}\right)}{\left(2 S_{k}\right)^{\lambda-1}} \frac{\sigma\left(2 S_{k}\right)}{\left(2 S_{k}\right)^{\lambda}} \leq \\
& \leq \pi\left(\frac{1}{\log 2}+\lambda\right) \frac{T\left(2 R_{k}, S\right)}{R_{k}^{\lambda}} .
\end{aligned}
$$

By Lemma 4 , for $k \geq k_{0}(\varepsilon)$ we obtain the inequality

$$
\int_{2 S_{k}}^{R_{k}} \frac{h(r)}{r^{\lambda+1}} d r<\varepsilon \int_{2 S_{k}}^{R_{k}} \frac{T(r, S)}{r^{\lambda+1}} d r .
$$

Therefore there exists a sequence $r_{k} \in\left[2 S_{k}, R_{k}\right]$ such that $h\left(r_{k}\right)<\varepsilon T\left(r_{k}, S\right)$. Moreover, from the definition of $h(r)$ if follows that there exists a sequence $r_{k} \rightarrow \infty$ such that for $k \geq k_{0}(\varepsilon)$

$$
\begin{gathered}
-\frac{p_{\phi}^{2}(\infty, S)}{\pi} u_{\phi}^{*}\left(r_{k}, \alpha\right) \cos \frac{\lambda(\alpha+\psi)}{p_{\phi}(\infty, S)}+\frac{p_{\phi}^{2}(\infty, S)}{\pi} \log \max _{|z|=r_{k}}\|\mathbf{x}(z)\| \cos \frac{\lambda \psi}{p_{\phi}(\infty, S)}+ \\
+\lambda p_{\phi}(\infty, S) N\left(r_{k}, \infty, S\right) \sin \frac{\lambda \psi}{p_{\phi}(\infty, S)}-\lambda p_{\phi}(\infty, S) T^{*}\left(r_{k}, \alpha, u_{\phi}\right) \sin \frac{\lambda \psi}{p_{\phi}(\infty, S)}<\varepsilon T\left(r_{k}, S\right) .
\end{gathered}
$$

Let us put $\psi=\frac{\pi p_{\phi}(\infty, S)}{2 \lambda}-\alpha$ in $(22)$. We obtain

$$
\begin{gathered}
\log \max _{|z|=r_{k}}\|\mathrm{x}(z)\| \sin \frac{\lambda \alpha}{p_{\phi}(\infty, S)}-\frac{\pi \lambda}{p_{\phi}(\infty, S)} T^{*}\left(k, \alpha, u_{\phi}\right)+ \\
+\frac{\pi \lambda}{p_{\phi}(\infty, S)} N\left(r_{k}, \infty, S\right) \cos \frac{\lambda \alpha}{p_{\phi}(\infty, S)}<\varepsilon T\left(r_{k}, S\right) .
\end{gathered}
$$

Since $T^{*}\left(r_{k}, \alpha, u_{\phi}\right) \leq T\left(r_{k}, S\right)+\phi\left(r_{k}\right)$ and $\frac{\lambda \alpha}{p_{\phi}(\infty, S)}<\frac{\pi}{2}$, we have

$$
\log \max _{|z|=r_{k}}\|\mathrm{x}(z)\| \sin \frac{\lambda \alpha}{p_{\phi}(\infty, S)}-\frac{\pi \lambda}{p_{\phi}(\infty, S)} T\left(r_{k}, S\right)<\varepsilon T\left(r_{k}, S\right) .
$$

Hence

$$
\beta(\infty, S) \leq \frac{\varepsilon+\frac{\pi \lambda}{p_{\phi}(\infty, S)}}{\sin \frac{\lambda \alpha}{p_{\phi}(\infty, S)}}
$$


where $\varepsilon>0$ and $\alpha, 0<\alpha \leq \min \left(\pi, \frac{\pi p_{\phi}(\infty, S)}{2 \lambda}\right)$, are chosen arbitrarily. Now we are able to prove the first and the second case of this theorem.

Let us consider the case of $\frac{\lambda}{p(\infty, S)} \geq \frac{1}{2}$. Then for all $\phi(r)$ we have $\frac{\lambda}{p_{\phi}(\infty, S)} \geq \frac{1}{2}$, which implies $\frac{\pi p_{\phi}(\infty, S)}{2 \lambda} \leq \pi$. Let in $(23) \alpha=\frac{\pi p_{\phi}(\infty, S)}{2 \lambda}$. Then $\beta(\infty, S) \leq \varepsilon+\frac{\pi \lambda}{p_{\phi}(\infty, S)}$.

In view of the arbitrariness of the number $\varepsilon$, we have $\beta(\infty, S) \leq \frac{\pi \lambda}{p_{\phi}(\infty, S)}$. This is true for each $\phi(r)$, hence by the definition of $p(\infty, S)$ we have $\beta(\infty, S) \leq \frac{\pi \lambda}{p(\infty, S)}$.

If $p(\infty, S)=1$ and $\lambda<\frac{1}{2}$ then there exists $\phi_{1}(r)$ such that $p_{\phi_{1}}(\infty, S)=1$. Therefore for each $\alpha, 0<\alpha<\pi$ we have $\beta(\infty, S) \leq \frac{\pi \lambda}{\sin \lambda \alpha}$. Thus $\beta(\infty, S) \leq \frac{\pi \lambda}{\sin \pi \lambda}$. Let now $\frac{\lambda}{p(\infty, S)}<\frac{1}{2}$ and $p(\infty, S)>1$. Then

$$
u_{\phi}^{*}(r, \pi)=\max \left(\min _{|z|=r} \log \|\mathrm{x}(z)\|, \phi(r)\right)=\phi(r)=o(T(r, S)) \quad(r \rightarrow \infty) .
$$

By substituting $\alpha=\pi$ and $\psi=0$ in the inequality (22) for $k \geq k_{0}$ we obtain

$$
\log \max _{|z|=r_{k}}\|\mathbf{x}(z)\|-\frac{\lambda \pi}{p_{\phi}(\infty, S)} T^{*}\left(r_{k}, \pi, u_{\phi}\right) \sin \frac{\pi \lambda}{p_{\phi}(\infty, S)}<\varepsilon T\left(r_{k}, S\right) .
$$

Hence

$$
\beta(\infty, S) \leq \frac{\pi \lambda}{p_{\phi}(\infty, S)} \sin \frac{\pi \lambda}{p_{\phi}(\infty, S)},
$$

but $\phi(r)$ was arbitrarily so $\beta(\infty, S) \leq \frac{\pi \lambda}{p(\infty, S)} \sin \frac{\pi \lambda}{p(\infty, S)}$.

5. Examples. We consider the surface $S(f)$ given by the relations

$$
\left\{\begin{array}{l}
x_{1}(z)=\operatorname{Re}\left[3 f(z)-f^{3}(z)\right] \\
x_{2}(z)=\operatorname{Re}\left[i\left(3 f(z)+f^{3}(z)\right)\right] \\
x_{3}(z)=\operatorname{Re}\left[3 f^{2}(z)\right]
\end{array}\right.
$$

where $f(z)$ is a meromorphic function of finite lower order $\lambda$. By [13] $S(f)$ is then a meromorphic minimal surface of finite lower order $\lambda$ and $\beta(\infty, S)=\beta(\infty, f)$. Let us first consider the Mittag-Leffer's function ([11]), i.e.

$$
E_{\rho}(z)=\sum_{k=0}^{\infty} \frac{z^{k}}{\Gamma\left(1+\frac{k}{\rho}\right)}, \quad 0<\rho<\infty .
$$

By [11, p. 86] we know that

$$
T\left(r, E_{\rho}(z)\right)= \begin{cases}\frac{1}{\pi \rho} r^{\rho}+o\left(r^{\rho}\right) & \text { for } \frac{1}{2} \leq \rho<\infty \\ \frac{\sin \pi \rho}{\pi \rho} r^{\rho}+o\left(r^{\rho}\right) & \text { for } 0<\rho<\frac{1}{2}\end{cases}
$$

To get the equality in the first case of Theorem 1 we take any $\lambda>0$ and $n \in \mathbb{N}$ such that $\frac{\lambda}{n}>\frac{1}{2}$ and consider the function $f_{1}(z)=E_{\frac{\lambda}{n}}\left(z^{n}\right)$. Then $T\left(r, f_{1}(z)\right)=\frac{n}{\pi \lambda} r^{\lambda}+o\left(r^{\lambda}\right)(r \rightarrow \infty)$.

Moreover, this function is entire and has $n$ maximum modulus points so $p\left(\infty, f_{1}\right)=n$. Note that $\mathcal{L}\left(r, \infty, f_{1}(z)\right)=r^{\lambda}+O(1)$, so $\mathcal{L}\left(r, \infty, f_{1}(z)\right) \sim r^{\lambda} \quad(r \rightarrow \infty)$. Hence we have $\beta\left(\infty, f_{1}(z)\right)=\frac{\pi \lambda}{n}$, so $\beta\left(\infty, S\left(f_{1}\right)\right)=\beta\left(\infty, f_{1}\right)=\frac{\pi \lambda}{n}$.

We should also note that by [13] $\log \|\mathbf{x}(z)\| \stackrel{n}{=} 3 \log \left|f_{1}(z)\right|+O(1)(r \rightarrow \infty)$ so by the definition of $p(\infty, S)$ we obtain $p\left(\infty, S\left(f_{1}\right)\right)=p\left(\infty, f_{1}\right)=n$. 
Thus the first estimate from Theorem 1 is attained for $S\left(f_{1}\right)$.

To prove the sharpness of the estimate in the second case we consider for each $0<\lambda \leq \frac{1}{2}$ the function $f_{2}(z)=E_{\lambda}(z)$.

We have $p\left(\infty, f_{2}\right)=1$. The function $f_{2}(z)$ is of finite lower order $\lambda$ and from (24) we have

$$
T\left(r, f_{2}(z)\right)=T\left(r, E_{\lambda}\right)=\frac{\sin \pi \lambda}{\pi \lambda} r^{\lambda}+o\left(r^{\lambda}\right), \quad \mathcal{L}\left(r, \infty, f_{2}(z)\right)=r^{\lambda}+O(1) \quad(r \rightarrow \infty) .
$$

Hence $\beta\left(\infty, f_{2}(z)\right)=\frac{\pi \lambda}{\sin \pi \lambda}$, thus

$$
\beta\left(\infty, S\left(f_{2}\right)\right)=\beta\left(\infty, f_{2}\right)=\frac{\pi \lambda}{\sin \pi \lambda} .
$$

Moreover, from the equality $\log \|\mathbf{x}(z)\|=3 \log \left|f_{2}(z)\right|+O(1)$ and from the definition of $p(\infty, S)$ we have

$$
p\left(\infty, S\left(f_{2}\right)\right)=p\left(\infty, f_{2}\right)=1,
$$

Thus the estimate from Theorem 1 in the second case is attained too.

\section{REFERENCES}

1. Baernstein A., Integral means, univalent functions and circular simmetrization, Acta Math., 133 (1974), 139-169.

2. Beckenbach E.F., Hutchison G.A., Meromorphic minimal surfaces, Pac. Journ. of Math., 28 (1969), 14-47.

3. Beckenbach E.F., Cootz T., The second fundamental theorem for meromorphic minimal surfaces, Bull. Amer. Math. Soc., 76 (1970), 711-716.

4. Blashke W., Differentialgeometrie und geometrische Grundlagen von Einsteins Relativitätstheorie. Russian transl., ONTI, Moscow, 1935, 703 p.

5. Ciechanowicz E., Marchenko I.I., Maximum modulus points, deviations and spreads of meromorphic functions, Value Distribution Theory and Related Topics, Kluwer, 2004, 117-129.

6. Ciechanowicz E., Marchenko I.I., On the maximum modulus points of entire and meromorphic functions, Mat. Stud., 21 (2004), №1, 25-34.

7. Ciechanowicz E., Marchenko I.I., On the separated maximum modulus points of meromorphic functions, Mat. fizika, analiz, geometriya, 12 (2005), №2, 218-229.

8. Ciechanowicz E., Marchenko I.I., A note on the separated maximum modulus points of meromorphic fuctions, Ann. Pol. Math., 110 (2014), №3, 295-310.

9. Essen M., Shea D.F., Applications of Denjoy integral inequalities and differential inequalities to growth problems for subharmonic and meromorphic functions, Proc. Roy. Irish Acad. Sect., A82 (1982), 201216.

10. Gariepy R., Lewis J.L., Space analogues of some theorems for subharmonic and meromorphic functions, Ark. Mat., 13 (1975), 91-105.

11. Goldberg A.A., Ostrovskii I.V., Distribution of values of meromophic functions, Nauka, Moscow, 1970, (in Russian); Engl. transl. : AMS Transl., 236, Providence, 2008.

12. Hayman W.K., Multivalent functions, Cambridge Univ. Press, Cambridge, 1958.

13. Marchenko I.I., Growth of meromorphic minimal surfaces, Teor. Funkts., Funkts. Anal., Prilozh., 34 (1979), 95-98. (in Russian)

14. Marchenko I.I., On magnitudes of deviations and spreads of meromorphic functions of fnite lower order, Mat. Sb., 186 (1995), 85-102, (in Russian); Engl. transl.: Sb. Mat., 186 (1995), 391-408.

15. Marchenko I.I., On the maximum modulus points of entire and meromorphic functions and the problem of Erdös, Mat. Stud., 38 (2012), №2, 212-215. 
16. Petrenko V.P., Growth of meromorphic functions. - Kharkov, Vyshcha Shkola, 1978, 136 p. (in Russian)

17. Petrenko V.P., Growth and distribution of values of minimal surfaces, Dokl. Akad. Nauk SSSR, 256 (1981), №1, 40-43. (in Russian)

18. Vekua I.N., The basics of tensor analysis and theory of covariants. - Nauka, Moscow, 1978. (in Russian)

School of Mathematics

West Pomeranian University of Technology, Szczecin, Poland

akowalski@zut.edu.pl

Faculty of Mathematics and Physics

University of Szczecin, Poland

iwan.marczenko@usz.edu.pl 